\title{
Multi-Higgs doublet models: the Higgs-fermion couplings and their sum rules
}

\author{
Miguel P. Bento, ${ }^{a}$ Howard E. Haber, $^{b}$ J.C. Romão ${ }^{a}$ and João P. Silva ${ }^{a}$ \\ ${ }^{a}$ CFTP, Departamento de Física, Instituto Superior Técnico, Universidade de Lisboa, \\ Avenida Rovisco Pais 1, Lisboa, 1049 Portugal \\ ${ }^{b}$ Santa Cruz Institute for Particle Physics, University of California, \\ 1156 High Street, Santa Cruz, California, 95064 U.S.A. \\ E-mail: miguelfilipebento@gmail.com, haber@scipp.ucsc.edu, \\ jorge.romao@tecnico.ulisboa.pt, jpsilva@cftp.tecnico.ulisboa.pt
}

ABSTRACT: This is the second of a series of papers that explores the physical parameterization, sum rules and unitarity bounds arising from a non-minimal scalar sector of the Standard Model (SM) that consists of $N$ Higgs doublets. In this paper, we focus on the structure and implication of the Yukawa interactions that couple the $N$ scalar doublets to the SM fermions. We employ the charged Higgs basis, which is defined as the basis of scalar fields such that the neutral scalar field vacuum expectation value resides entirely in one of the $N$ scalar doublet fields, and the charged components of the remaining $N-1$ scalar doublet fields are the physical (mass-eigenstate) charged Higgs fields. Based on the structure of the Yukawa Lagrangian of the model (and as a consequence of tree-level unitarity), one may deduce numerous sum rules, several of which have not appeared previously in the literature. These sum rules can be used to uncover intimate relations between the structure of the Higgs-fermion couplings and the scalar/gauge couplings. In particular, we show that the approximate alignment limit, in which the $W^{+} W^{-}$and $Z Z$ couplings to the observed Higgs boson are approximately SM-like, imposes significant constraints on the Higgs-fermion couplings.

Keywords: Beyond Standard Model, Higgs Physics

ArXiv EPrint: 1808.07123 


\section{Contents}

1 Introduction 1

2 The $N$ Higgs doublet model with fermions 2

$\begin{array}{lll}3 & \text { Sum rules } & 9\end{array}$

4 A critical constraint from perturbativity 12

5 Conclusions $\quad 14$

$\begin{array}{ll}\text { A Generalized sum rules } & \mathbf{1 5}\end{array}$

$\begin{array}{lll}\text { A.1 Notation and conventions } & 15\end{array}$

A.2 FFVV sum rules $\quad 16$

$\begin{array}{lll}\text { A.2.1 The amplitudes } & 16\end{array}$

$\begin{array}{ll}\text { A.2.2 The high energy limit } & 16\end{array}$

A.3 FFV sum rules 20

$\begin{array}{lll}\text { A.3.1 The amplitudes } & 20\end{array}$

A.3.2 The high energy limit 20

\section{Introduction}

The discovery of the Higgs boson at the Large Hadron Collider (LHC) at CERN [1-3] leaves two crucial open questions. First, how many elementary scalars there are in Nature? Is there one single scalar as in the original proposal for the Standard Model (SM), or are there several scalar families, just like there are several families of elementary fermions? Second, are the couplings of the observed $125 \mathrm{GeV}$ scalar to gauge bosons and to fermions consistent with the SM (and if yes, to what precision)? Answering this second question will place important constraints on models of new physics beyond the SM. The two questions posed above are related. For example, a detailed study of the predictions of an $N$ Higgs doublet model (NHDM) can guide experimental searches for new scalar phenomena.

Models with multiple scalar doublets are very rich. They predict both neutral and charged scalars, whose mass basis do not, in general, coincide with the interaction basis. As a result, one has mixing among the neutral scalars and mixing among the charged scalars. In addition, new sources of $\mathrm{CP}$ violation in the scalar sector are possible, and the mechanism for $\mathrm{CP}$ violation may be spontaneous [4] or explicit. One might have new $\mathrm{CP}$ violation sources in the mixing of neutral CP-even and CP-odd scalars, in the mixing of charged scalars, and/or in the couplings of scalars with fermions. In general, the NHDM also yields flavor changing neutral scalar interactions, which are strongly constrained by experiment. 
This difficulty is a challenge, which one can address with extra symmetries, such as the $\mathbb{Z}_{2}$ symmetry introduced in the two Higgs doublet model (2HDM) following general theorems proposed by Glashow and Weinberg [5] and independently by Paschos [6]. However, the difficulty in avoiding flavor changing interactions mediated by neutral scalars is also an opportunity. For example, the symmetries employed in suppressing flavor changing neutral scalar interactions might also be related to the hierarchy of fermion masses and mixing, or even to the existence of Dark Matter.

In order to study models of extended Higgs sectors, one needs first to establish a convenient notation and impose the relevant theoretical constraints, such as those arising from unitarity bounds. In a previous publication [7], we introduced a suitable notation in the pure scalar sector of the NHDM, clearly related to the physical degrees of freedom, which we identified as being those that appear in the mass basis of the charged scalars, and we studied the unitarity bounds arising from the scalar/gauge sectors. Here, we will extend our parameterization into the fermion sector, and we will study the unitarity bounds arising from interaction of the fermions with the scalar/gauge sectors.

Section 2 reviews and extends our physical parameterization and the many relations among the parameters. These are used in section 3 in order to derive new sum rules. In section 4 we define a vector involving the couplings in the gauge/scalar sector and vectors involving the Yukawa couplings. We show that the approximate Higgs alignment observed in the gauge/scalar sector (where the properties of one neutral scalar is SM-like) translates into an alignment between the gauge/scalar sector vector and vectors in the scalar/fermion sector. We present our conclusions in section 5. In appendix A, we use the cancellation of bad high energy behavior in $2 \rightarrow 2$ scattering amplitudes in order to rederive the sum rules that have been obtained in section 3 by looking directly at the Lagrangian.

\section{The $N$ Higgs doublet model with fermions}

In this section we discuss the full Lagrangian of the most general $N$ Higgs doublet model. Our field content is the following: (i) The usual $\mathrm{SU}(2)_{L} \times \mathrm{U}(1)_{Y}$ gauge bosons; (ii) $N$ Higgs doublet fields, parameterized as:

$$
\Phi_{k}=\left(\begin{array}{c}
\varphi_{k}^{+} \\
\frac{1}{\sqrt{2}}\left(v_{k}+\varphi_{k}^{0}\right)
\end{array}\right),
$$

for $k=1,2, \ldots, N$; and (iii) the quark doublets, $q_{L}=\left(p_{L}, n_{L}\right)$, which is a vector in the $n_{g}$-dimensional family space of left-handed doublets, and the quark singlets, $p_{R}$ and $n_{R}$, which are $n_{g}$-dimensional vectors in the right-handed family spaces of charge $2 / 3$ and $-1 / 3$ quarks, respectively. ${ }^{1}$ The neutral scalar field vacuum expectation values are normalized such that

$$
v^{2} \equiv v_{1}^{2}+v_{2}^{2}+\ldots v_{N}^{2}=(246 \mathrm{GeV})^{2},
$$

whose numerical value is fixed by the Fermi constant.

\footnotetext{
${ }^{1}$ Throughout this paper we neglect leptons without loss of generality, as our analysis of the quark sector is similar to that of the lepton sector with Dirac neutrinos.
} 
When expressed in terms of the physical gauge fields, the covariant derivative may be written as

$$
i D_{\mu}=i \partial_{\mu}-\frac{g}{2}\left(\tau_{+} W_{\mu}^{+}+\tau_{-} W_{\mu}^{-}\right)-e Q A_{\mu}-\frac{g}{c_{W}}\left(\frac{\tau_{3}}{2}-Q s_{W}^{2}\right) Z_{\mu}
$$

where $g$ is the $\mathrm{SU}(2)$ coupling constant, $c_{W}=\cos \theta_{W}, s_{W}=\sin \theta_{W}, e$ is the positron charge, $Q$ is the charge operator, and the $\mathrm{SU}(2)$ generators, when acting on doublets,

$$
\tau_{+}=\left(\begin{array}{cc}
0 & \sqrt{2} \\
0 & 0
\end{array}\right), \quad \tau_{-}=\left(\begin{array}{cc}
0 & 0 \\
\sqrt{2} & 0
\end{array}\right), \quad \tau_{3}=\left(\begin{array}{rr}
1 & 0 \\
0 & -1
\end{array}\right) .
$$

The covariant derivative for the singlet right-handed quarks only contains $i \partial_{\mu}$ and the terms proportional to $Q$ in eq. (2.3). Our choice for the signs of the coupling constants and of the gauge fields is that in ref. [8] with all $\eta$ factors taken positive. The kinetic terms are written as

$$
\begin{aligned}
\mathcal{L}_{K \Phi} & =\left(D_{\mu} \Phi_{k}\right)^{\dagger}\left(D_{\mu} \Phi_{k}\right), \\
\mathcal{L}_{K q} & =\bar{q}_{L}(i \not D) q_{L}+\bar{p}_{R}(i \not D) p_{R}+\bar{n}_{R}(i \not D) n_{R},
\end{aligned}
$$

for the scalars and quarks, respectively.

For the scalar potential, we follow the notation of $[9,10]$ :

$$
V_{H}=\mu_{i j}\left(\Phi_{i}^{\dagger} \Phi_{j}\right)+\lambda_{i j, k l}\left(\Phi_{i}^{\dagger} \Phi_{j}\right)\left(\Phi_{k}^{\dagger} \Phi_{l}\right)=-\mathcal{L}_{\mathrm{Higgs}},
$$

where, by hermiticity,

$$
\mu_{i j}=\mu_{j i}^{*}, \quad \lambda_{i j, k l} \equiv \lambda_{k l, i j}=\lambda_{j i, l k}^{*} .
$$

The Yukawa couplings are organized into complex $n_{g} \times n_{g}$ matrices $\Gamma_{k}$ and $\Delta_{k}$ (for $k=1, \ldots, N)$, as

$$
-\mathcal{L}_{Y}=\bar{q}_{L} \Gamma_{k} \Phi_{k} n_{R}+\bar{q}_{L} \Delta_{k} \tilde{\Phi}_{k} p_{R}+\text { h.c. }
$$

where $\tilde{\Phi}_{k} \equiv i \tau_{2} \Phi_{k}^{*}$.

Under a weak basis transformation of the scalars, ${ }^{2}$

$$
\Phi_{j}=X_{j b} \Phi_{b}^{\prime}
$$

the couplings with scalars transform into

$$
\begin{aligned}
\mu_{a b}^{\prime} & =X_{i a}^{*} \mu_{i j} X_{j b}, \\
\lambda_{a b, c d}^{\prime} & =X_{i a}^{*} X_{k c}^{*} \lambda_{i j, k l} X_{j b} X_{l d}, \\
\Gamma_{b}^{\prime} & =\Gamma_{j} X_{j b}, \\
\Delta_{b}^{\prime} & =\Delta_{i} X_{i b}^{*} .
\end{aligned}
$$

In a previous publication [7] we stressed the importance of the charged Higgs basis, defined as the basis where the charged components of all scalar doublets correspond to

\footnotetext{
${ }^{2} \mathrm{~A}$ weak basis transformation is one which preserves the doublet structure.
} 
charged scalar mass eigenstates [11]. We may parameterize the fields in the charged Higgs basis as

$$
\Phi_{1}^{C}=\left(\begin{array}{c}
G^{+} \\
\frac{1}{\sqrt{2}}\left(v+H^{0}+i G^{0}\right)
\end{array}\right), \quad \Phi_{2}^{C}=\left(\begin{array}{c}
S_{2}^{+} \\
\frac{1}{\sqrt{2}} \varphi_{2}^{C 0}
\end{array}\right), \quad \ldots, \quad \Phi_{N}^{C}=\left(\begin{array}{c}
S_{N}^{+} \\
\frac{1}{\sqrt{2}} \varphi_{N}^{C 0}
\end{array}\right),
$$

where $S_{1}^{+} \equiv G^{+}$is he charged massless would-be Goldstone boson and $S_{2}^{+}, \ldots, S_{N}^{+}$are the physical (mass-eigenstate) charged Higgs fields, with corresponding masses $m_{ \pm, a}^{2}$. Notice that only the neutral component of the first doublet has a vacuum expectation value. In ref. [7], it is shown that all scalar-scalar and scalar-gauge couplings depend exclusively on a single $N \times 2 N$ matrix $B$. Its physical significance is the matrix that takes the neutral scalars fields from the charged Higgs basis into their mass eigenstate basis. Denoting $\varphi_{1}^{C 0} \equiv H^{0}+i G^{0}$, the neutral Higgs fields in the charged Higgs basis are given in terms of the neutral mass-eigenstate scalar fields,

$$
\varphi_{a}^{C 0}=\sum_{\beta=1}^{2 N} B_{a \beta} S_{\beta}^{0},
$$

where $S_{1}^{0} \equiv G^{0}$ is the neutral massless would-be Goldstone boson and $S_{2}^{0}, \ldots, S_{2 N}^{0}$ are the physical (mass-eigenstate) neutral Higgs scalar fields, with corresponding masses $m_{\beta}^{2}$. We may therefore introduce a basis transformation $X=U$, where $U$ is a unitary matrix that diagonalizes the charged scalar squared-mass matrix such that

$$
\varphi_{k}^{+}=\sum_{a=1}^{N} U_{k a} S_{a}^{+}
$$

with the corresponding diagonal charged scalar squared-mass matrix denoted by

$$
D_{ \pm}^{2} \equiv \operatorname{diag}\left(m_{ \pm, 1}^{2}=0, m_{ \pm, 2}^{2}, \ldots, m_{ \pm, N}^{2}\right),
$$

and $U_{k 1}=v_{k} / v$.

In the charged Higgs basis, the neutral fields $\varphi_{a}^{C 0}$ are related to the neutral scalar fields of the original basis defined in eq. (2.1),

$$
\varphi_{k}^{0}=\sum_{a=1}^{N} U_{k a} \varphi_{a}^{C 0}=\sum_{\beta=1}^{2 N} \sum_{a=1}^{N} U_{j a} B_{a \beta} S_{\beta}^{0} .
$$

Note that one can also diagonalize the neutral scalar squared-mass matrix starting from the original basis of scalar fields,

$$
\varphi_{k}^{0}=\sum_{\beta=1}^{2 N} V_{k \beta} S_{\beta}^{0}
$$

with the corresponding diagonal neutral scalar squared-mass matrix denoted by

$$
D_{0}^{2} \equiv \operatorname{diag}\left(m_{1}^{2}=0, m_{2}^{2}, \ldots, m_{2 N}^{2}\right),
$$


and $V_{k 1}=i v_{k} / v$. Eqs. (2.18) and (2.19) imply that,

$$
B=U^{\dagger} V .
$$

It is straightforward to see that $U^{\dagger}$ is the matrix that takes the scalar doublets from the original basis to a charged Higgs basis. Because the latter is defined up to a rephasing of $N-1$ doublets [7], the transformation into this basis is not unique. For example, one could consider a matrix $U^{\prime}=U K$ where $^{3}$

$$
K=\operatorname{diag}\left(1, e^{-i \chi_{2}}, e^{-i \chi_{3}}, \cdots, e^{-i \chi_{N}}\right) .
$$

Furthermore, because $U$ is a basis transformation, it is parameterized by $N^{2}$ non-physical parameters. It is then easy to see that the matrix $B$ alone comprises all the relevant physical parameters of the diagonalization of the charged and neutral scalar fields.

The non-uniqueness of the charged Higgs basis implies that the matrix $U$ employed in eq. (2.16) can be replaced by $U^{\prime}=U K$. That is $U_{k 1}^{\prime}=U_{k 1}$ and $U_{j a}^{\prime}=e^{-i \chi_{a}} U_{j a}$ for $a=2,3, \ldots, N$. Eq. (2.21) then yields,

$$
B_{1 \beta}^{\prime}=B_{1 \beta}, \quad B_{a \beta}^{\prime}=e^{i \chi_{a}} B_{a \beta}, \quad \text { for } a=2,3, \ldots, N .
$$

The unphysical phases $\chi_{a}$ can be absorbed into the definition of the charged Higgs basis scalar doublet fields, $\Phi_{a}^{C}$. That is,

$$
\Phi_{a}^{C} \rightarrow e^{i \chi_{a}} \Phi_{a}^{C}, \quad \text { for } a=2,3, \ldots, N .
$$

In particular, note that under this rephasing, the charged Higgs fields rephase in the same way, i.e., $S_{a}^{+} \rightarrow e^{i \chi_{a}} S_{a}^{+}$(for $a=2,3, \ldots, N$ ). In contrast, the mass eigenstate neutral Higgs fields $S_{\beta}^{0}$ are invariant under this rephasing in light of eq. (2.18) since the rephasing of $\phi_{a}^{C 0}$ is consistent with the rephasing of $B_{a \beta}$.

In defining the neutral Higgs mass eigenstate fields, $S_{\beta}^{0}$, one always has the option to redefine any of the neutral scalar fields via $S_{\beta}^{0} \rightarrow-S_{\beta}^{0}$. This means that the choice of the matrix elements of the diagonalization matrix in eq. (2.19) is unique only up to a sign, $V_{k \beta} \rightarrow-V_{k \beta}$. That is, one is free to change the overall sign of any column of $V$. For example, taking $h=S_{2}^{0}$ to be the $125 \mathrm{GeV}$ neutral Higgs field, the overall signs of the couplings, $\operatorname{sgn}[h V V]$ and $\operatorname{sgn}[h b b]$, do not have physical significance, although the relative sign of these two couplings is physical and can be measured. ${ }^{4}$ It is common practice to employ a specific sign convention to uniquely fix the signs of the neutral Higgs mass eigenstate fields. In this convention, $V_{k 1}=i v_{k} / v$ and the $V_{k j}$ (for $j=2,3, \ldots, 2 N$ ) are parameterized by $(N-1)(2 N-1)$ real angles $\theta_{k \ell}$ (for $\left.1 \leq k<\ell \leq 2 N-1\right)$ [13]. The ranges of the $\theta_{k \ell}$ can then be chosen to uniquely fix the signs of the columns of $V$ [14].

\footnotetext{
${ }^{3}$ Here, we shall assume that there are no mass degeneracies among the charged Higgs bosons. If mass degeneracies exist, then the most general form for $K$ would be a block diagonal form with an $n \times n$ unitary matrix replacing a diagonal matrix of phases within the $n$-dimensional mass-degenerate subspace. For further details, see ref. [12].

${ }^{4}$ This is the source of some confusion in the literature, even in the 2HDM.
} 
In ref. [7], the following properties of the $N \times 2 N$ matrix $V$ were obtained,

$$
\begin{aligned}
\operatorname{Re}\left(V^{\dagger} V\right) & =\mathbb{1}_{2 N \times 2 N}, \\
V V^{\dagger} & =2 \cdot \mathbb{1}_{N \times N}, \\
V V^{T} & =0 .
\end{aligned}
$$

Several properties of $B$ have been thoroughly studied in ref. [7], extending previous work in refs. [15-18]. For example, in light of eq. (2.21) and using the fact that $U$ is unitary, eqs. (2.25)-(2.27) yield,

$$
\begin{aligned}
\frac{1}{2} B B^{\dagger} & =\mathbb{1}_{N \times N}, \\
\operatorname{Re}\left(B^{\dagger} B\right) & =\mathbb{1}_{2 N \times 2 N}, \\
B B^{T} & =0 .
\end{aligned}
$$

We may also define a new orthogonal and antisymmetric matrix

$$
A=\operatorname{Im}\left(B^{\dagger} B\right)
$$

which appears in gauge boson couplings to two neutral scalars. Using eq. (2.29), one can write,

$$
B^{\dagger} B=\mathbb{1}_{2 N \times 2 N}+i A .
$$

From eq. (2.31), it immediately follows that,

$$
A^{T}=-A, \quad A A^{T}=-A^{2}=\mathbb{1}_{2 N \times 2 N},
$$

after employing eqs. (2.28)-(2.30). Furthermore, after multiplying eq. (2.32) on the left by $B$ and using eq. (2.28), we obtain

$$
B A=-i B
$$

Eq. (2.34), which is stated here explicitly for the first time, plays a significant role in the intermediate steps of the calculations employed later in this work. Finally, we note the following explicit relations previously obtained in ref. [7],

$$
\begin{aligned}
& B_{a 1}=i \delta_{a 1}, \\
& B_{1 \beta}=-A_{1 \beta}+i \delta_{1 \beta} .
\end{aligned}
$$

Eq. (2.35) is just the statement that $S_{1}^{0}=G^{0}$ resides entirely in the imaginary part of $\varphi_{1}^{C 0}$. Note that eq. (2.36) can be obtained by using the relation, $A_{1 \beta}=-\operatorname{Re} B_{1 \beta}$, which is a consequence of eqs. (2.31) and (2.35).

In the charged Higgs basis, the scalar potential takes the following form,

$$
\mathcal{V}_{H}=Y_{a b}\left(\Phi_{a}^{C \dagger} \Phi_{b}^{C}\right)+Z_{a b, c d}\left(\Phi_{a}^{C \dagger} \Phi_{b}^{C}\right)\left(\Phi_{c}^{C \dagger} \Phi_{d}^{C}\right) .
$$

The minimization the scalar potential in the charged Higgs basis and the identification of the charged Higgs boson squared-masses are neatly summarized by the following equation obtained in ref. [7],

$$
Y_{a b}+v^{2} Z_{a b, 11}=\left(D_{ \pm}^{2}\right)_{a b}
$$


In addition, there are a number of notable relations among the squared-masses of the neutral and charged scalars, the physical mixing matrices $(A$ and $B)$, and the coefficients of the scalar potential in the charged Higgs basis $(Y$ and $Z)$. For example, performing the diagonalization of the neutral scalar squared-mass directly in the charged Higgs basis yields [7],

$$
\begin{aligned}
& 2 v^{2} Z_{i 1,1 j}=-2\left(D_{ \pm}^{2}\right)_{i j}+\left(B D_{0}^{2} B^{\dagger}\right)_{i j}, \\
& 2 v^{2} Z_{i 1, j 1}=\left(B D_{0}^{2} B^{T}\right)_{i j} .
\end{aligned}
$$

Using these results along with eqs. (2.36) and (2.38), one can easily derive,

$$
\begin{aligned}
Y_{1 a} & =\frac{1}{2}\left(A D_{0}^{2} B^{\dagger}\right)_{1 a}, \\
Y_{a 1} & =-\frac{1}{2}\left(B D_{0}^{2} A\right)_{a 1} .
\end{aligned}
$$

In the charged Higgs basis, the Yukawa Lagrangian takes the following form,

$$
-\mathcal{L}_{Y}=\bar{q}_{L} \Gamma_{a}^{C} \Phi_{a}^{C} n_{R}+\bar{q}_{L} \Delta_{a}^{C} \tilde{\Phi}_{a}^{C} p_{R}+\text { h.c. },
$$

where

$$
\tilde{\Phi}_{a}^{C} \equiv\left(\begin{array}{c}
\frac{1}{\sqrt{2}}\left(\varphi_{a}^{C 0}\right)^{*} \\
-S_{a}^{-}
\end{array}\right) .
$$

The quarks are brought into their mass basis by unitary transformations

$$
\begin{array}{ll}
n_{R}=U_{d R} d_{R}, & p_{R}=U_{u R} u_{R}, \\
n_{L}=U_{d L} d_{L}, & p_{L}=U_{u L} u_{L} .
\end{array}
$$

Since only the neutral component of the first doublet $\Phi^{C}$ has a vacuum expectation, these transformations are chosen such that

$$
\begin{aligned}
\frac{v}{\sqrt{2}} U_{d L}^{\dagger} \Gamma_{1}^{C} U_{d R}=D_{d} & =\operatorname{diag}\left(m_{d}, m_{s}, m_{b}, \ldots\right), \\
\frac{v}{\sqrt{2}} U_{u L}^{\dagger} \Delta_{1}^{C} U_{u R}=D_{u} & =\operatorname{diag}\left(m_{u}, m_{c}, m_{t}, \ldots\right) .
\end{aligned}
$$

The Yukawa Lagrangian can then be rewritten as,

$$
\begin{aligned}
-\frac{v}{\sqrt{2}} \mathcal{L}_{Y}= & \left(\bar{u}_{L} V, \bar{d}_{L}\right)\left(D_{d} \Phi_{1}^{C}+N_{d}^{(2)} \Phi_{2}^{C}+\cdots+N_{d}^{(N)} \Phi_{N}^{C}\right) d_{R} \\
& +\left(\bar{u}_{L}, \bar{d}_{L} V^{\dagger}\right)\left(D_{u} \tilde{\Phi}_{1}^{C}+N_{u}^{(2)} \tilde{\Phi}_{2}^{C}+\cdots+N_{u}^{(N)} \tilde{\Phi}_{N}^{C}\right) u_{R}+\text { h.c. }
\end{aligned}
$$

where $V=U_{u L}^{\dagger} U_{d L}$ is the Cabibbo-Kobayashi-Maskawa (CKM) matrix, and

$$
\begin{aligned}
& N_{d}^{(a)}=\frac{v}{\sqrt{2}} U_{d L}^{\dagger} \Gamma_{a}^{C} U_{d R}, \\
& N_{u}^{(a)}=\frac{v}{\sqrt{2}} U_{u L}^{\dagger} \Delta_{a}^{C} U_{u R},
\end{aligned}
$$




\begin{tabular}{|c|c|c|c|}
\hline \multicolumn{2}{|c|}{ Kinetic Lagrangian } & \multicolumn{2}{|c|}{ Yukawa Lagrangian } \\
\hline Coupling & Feynman rule & Coupling & Feynman rule \\
\hline$\left[\bar{u}_{n} d_{m} W_{\mu}^{+}\right]$ & $-i \frac{g}{\sqrt{2}} \gamma_{\mu} P_{L} V_{n m}$ & {$\left[\bar{u}_{n} d_{m} G^{+}\right]$} & $i \frac{g}{\sqrt{2}}\left(\frac{m_{n}^{u}}{M_{W}} P_{L}-\frac{m_{m}^{d}}{M_{W}} P_{R}\right) V_{n m}$ \\
\hline$\left[\bar{d}_{n} u_{m} W_{\mu}^{-}\right]$ & $-i \frac{g}{\sqrt{2}} \gamma_{\mu} P_{L} V_{n m}^{*}$ & {$\left[\bar{d}_{n} u_{m} G^{-}\right]$} & $i \frac{g}{\sqrt{2}}\left(\frac{m_{n}^{u}}{M_{W}} P_{R}-\frac{m_{m}^{d}}{M_{W}} P_{L}\right) V_{n m}^{*}$ \\
\hline$\left[\bar{u}_{n} u_{m} Z_{\mu}\right]$ & $-i \frac{g}{c_{w}} \gamma_{\mu}\left(\frac{1}{2} P_{L}-\frac{2}{3} s_{w}^{2}\right) \delta_{n m}$ & {$\left[\bar{u}_{n} u_{m} G^{0}\right]$} & $-\frac{g}{2 M_{W}} m_{n}^{u} \delta_{n m} \gamma_{5}$ \\
\hline$\left[\bar{d}_{n} d_{m} Z_{\mu}\right]$ & $i \frac{g}{c_{w}} \gamma_{\mu}\left(\frac{1}{2} P_{L}-\frac{1}{3} s_{w}^{2}\right) \delta_{n m}$ & {$\left[\bar{d}_{n} d_{m} G^{0}\right]$} & $\frac{g}{2 M_{W}} m_{n}^{d} \delta_{n m} \gamma_{5}$ \\
\hline
\end{tabular}

Table 1. The couplings of the up-type and down-type fermions to the massive gauge bosons and their Yukawa Lagrangian counterparts obtained by substituting the gauge bosons by the corresponding Goldstone bosons.

with $a=2,3, \ldots, N$. In light of eq. (2.24), the matrices $N_{d}^{(a)}$ and $N_{u}^{(a)}$ rephase under the rephasing of the charged Higgs basis,

$$
N_{d}^{(a)} \rightarrow e^{-i \chi_{a}} N_{d}^{(a)}, \quad N_{u}^{(a)} \rightarrow e^{i \chi_{a}} N_{u}^{(a)}, \quad \text { for } a=2,3, \ldots, N .
$$

In general, the matrices $N_{d}^{(a)}$ and $N_{u}^{(a)}$ are not diagonal, leading to flavor-changing neutral scalar interactions, which are strongly constrained experimentally. Notice that these matrices, multiplied by the appropriate CKM matrix element, will also be responsible for the charged scalar interactions with quarks. Using eqs. (2.15) and (2.44), it follows that

$$
\begin{aligned}
-\frac{v}{\sqrt{2}} \mathcal{L}_{Y}= & \bar{u}_{L} V\left(N_{d}^{(a)} S_{a}^{+}\right) d_{R}+\frac{v}{\sqrt{2}} \bar{d}_{L} D_{d} d_{R}+\frac{1}{\sqrt{2}} \bar{d}_{L}\left(N_{d}^{(a)} B_{a \beta} S_{\beta}^{0}\right) d_{R} \\
& -\bar{d}_{L} V^{\dagger}\left(N_{u}^{(a)} S_{a}^{-}\right) u_{R}+\frac{v}{\sqrt{2}} \bar{u}_{L} D_{u} u_{R}+\frac{1}{\sqrt{2}} \bar{u}_{L}\left(N_{u}^{(a)} B_{a \beta}^{*} S_{\beta}^{0}\right) u_{R}+\text { h.c. },
\end{aligned}
$$

where there is an implicit sum over repeated indices, $a=1, \ldots, N$ and $\beta=1, \ldots, 2 N$, and

$$
N_{d}^{(1)}=D_{d}, \quad N_{u}^{(1)}=D_{u},
$$

are identified as the diagonal down-type and up-type fermion mass matrices defined in eqs. (2.47) and (2.48). Note that eq. (2.53) includes the fermion interactions with the charged and neutral Goldstone bosons, $S_{1}^{+}=G^{+}$and $S_{1}^{0}=G^{0}$. Moreover, eq. (2.53) is invariant under the rephasing of the charged Higgs basis in light of eqs. (2.23) and (2.52).

The matrices $B$ and $N^{(a)}$ fully parameterize the Yukawa Lagrangian. Thus, we may use the equivalence theorem [19] in order to relate some of the cubic couplings from the kinetic Lagrangian in eq. (2.5) to the couplings with Goldstone bosons in eq. (2.53), through the properties of $B$ in eqs. (2.35) and (2.36). In contrast with our previous publication [7], where both the scalar potential and the kinetic Lagrangian Feynman rules were in general distinct from the SM, here we find that the fermion-gauge couplings of the NHDM are identical to those of the SM. These results are presented in table 1 , where $m_{n}^{u}\left(m_{n}^{d}\right)$ is the $n$-th up-quark (down-quark) mass. Thus, no new sum rules arise exclusively from the fermion-gauge couplings. 


\section{Sum rules}

A comprehensive study of sum rules for Higgs couplings in extended Higgs sectors (under the assumption of a CP-conservation) was first provided in ref. [20]. In ref. [7], we specialized to the NHDM (while relaxing the assumption of CP conservation in the scalar sector) and derived numerous sum rules involving the Higgs couplings in the scalar-gauge sector of the model (see also refs. [21, 22]). In this section, we extend our study of the NHDM sum rules to include the Higgs couplings to fermions.

We use the same notation of ref. [7] in which $\left[X_{a} Y_{b} Z_{c}\right]$ is identified as the term in the Lagrangian that depends explicitly on family type indices. For example [7], from the Feynman rules

$$
\begin{aligned}
Z_{\mu} S_{\beta}^{0} S_{\gamma}^{0}: & \frac{g}{2 c_{W}}\left(p_{\beta}^{0}-p_{\gamma}^{0}\right)_{\mu} A_{\beta \gamma}, \\
Z_{\mu} Z_{\nu} S_{\beta}^{0}: & -\frac{i g M_{Z}}{c_{W}} A_{1 \beta} g_{\mu \nu}, \\
W_{\mu}^{+} W_{\nu}^{-} S_{\beta}^{0}: & -i g M_{W} A_{1 \beta} g_{\mu \nu}, \\
W^{+} S_{a}^{-} S_{\beta}^{0}: & \frac{i g}{2}\left(p_{a}^{-}-p_{\beta}^{0}\right)^{\mu} B_{a \beta},
\end{aligned}
$$

we define

$$
\begin{aligned}
{\left[Z_{\mu} S_{\beta}^{0} S_{\gamma}^{0}\right] } & =A_{\beta \gamma}, & {\left[Z_{\mu} Z_{\nu} S_{\beta}^{0}\right] } & =A_{1 \beta}, \\
{\left[W_{\mu}^{+} W_{\nu}^{-} S_{\beta}^{0}\right] } & =A_{1 \beta}, & {\left[W^{+} S_{a}^{-} S_{\beta}^{0}\right] } & =B_{a \beta} .
\end{aligned}
$$

Since the matrix $A$ is antisymmetric, $A_{\beta \beta}=0$ whenever the two indices coincide.

Analogously, we define $\left[X_{a} Y_{b} Z_{c}\right]_{R, L}$ as the term that depends on family type indices that is proportional to the corresponding chiral projection operator $P_{R, L} \equiv \frac{1}{2}\left(1 \pm \gamma_{5}\right)$. For example, in the Lagrangian term

$$
\mathcal{L} \supset C_{1}\left\{f(a, b, c) P_{L}+g(a, b, c) P_{R}\right\} X_{a} Y_{b} Z_{c},
$$

involving the fields $X_{a}, Y_{b}, Z_{c}$, we identify $\left[X_{a} Y_{b} Z_{c}\right]_{L}=f(a, b, c)$ and $\left[X_{a} Y_{b} Z_{c}\right]_{R}=g(a, b, c)$. We employ indices $a$ and $\beta$ for scalars and indices $m, n, p$ and $q$ as fermion family indices, and we follow closely the sign conventions of ref. [8]. For convenience, we have extracted a normalization factor $C_{1}$, whose value depends on whether the scalar field is electrically charged or neutral. As an example, for the couplings of the charged scalars to fermion pairs, it is convenient to define $C_{1}=\sqrt{2} / v=g /\left(\sqrt{2} m_{W}\right)$. Then, ${ }^{5}$

$$
\begin{aligned}
{\left[\bar{u}_{n} d_{m} S_{a}^{+}\right]_{L} } & =\left(N_{u}^{\dagger(a)}\right)_{n p} V_{p m}, \\
{\left[\bar{u}_{n} d_{m} S_{a}^{+}\right]_{R} } & =-V_{n p}\left(N_{d}^{(a)}\right)_{p m},
\end{aligned}
$$

\footnotetext{
${ }^{5}$ One can check that eqs. (3.4)-(3.7) and eqs. (3.8)-(3.11) with the respective choices for $C_{1}$ are consistent by comparing these couplings for $\beta=1$ [cf. eq. (2.35)] with the SM couplings of the charged and neutral Goldstone boson to corresponding quark-antiquark pairs [23].
} 


$$
\begin{aligned}
{\left[\bar{d}_{n} u_{m} S_{a}^{-}\right]_{L} } & =-\left(N_{d}^{\dagger(a)}\right)_{n p}\left(V^{\dagger}\right)_{p m}, \\
{\left[\bar{d}_{n} u_{m} S_{a}^{-}\right]_{R} } & =\left(V^{\dagger}\right)_{n p}\left(N_{u}^{(a)}\right)_{p m}
\end{aligned}
$$

where repeated indices are summed. For the couplings of the neutral scalars to fermion pairs, it is convenient to define $C_{1}=-1 / v=-g /\left(2 m_{W}\right)$. Then, ${ }^{5}$

$$
\begin{aligned}
{\left[\bar{d}_{n} d_{m} S_{\beta}^{0}\right]_{L} } & =\left(N_{d}^{\dagger(a)}\right)_{n m}\left(B^{\dagger}\right)_{\beta a}, \\
{\left[\bar{d}_{n} d_{m} S_{\beta}^{0}\right]_{R} } & =\left(N_{d}^{(a)}\right)_{n m} B_{a \beta}, \\
{\left[\bar{u}_{n} u_{m} S_{\beta}^{0}\right]_{L} } & =\left(N_{u}^{\dagger(a)}\right)_{n m} B_{a \beta}, \\
{\left[\bar{u}_{n} u_{m} S_{\beta}^{0}\right]_{R} } & =\left(N_{u}^{(a)}\right)_{n m}\left(B^{\dagger}\right)_{\beta a} .
\end{aligned}
$$

In light of eqs. (2.49)-(2.51), the matrices $N_{f}^{(a)}$ and the couplings defined here have dimensions of mass. Once again, one can verify that all Yukawa interactions are independent of the rephasing of the charged Higgs basis (taking into account the corresponding rephasing of the charged Higgs fields, $S_{a}^{ \pm}$).

Based on the structure of the Yukawa Lagrangian of the NHDM, one may deduce several sum rules that have not appeared previously in the literature. For example,

$$
\begin{aligned}
& \sum_{\beta=1}^{2 N}\left[\bar{f}_{n} f_{m} S_{\beta}^{0}\right]_{L}\left[\bar{f}_{p} f_{q} S_{\beta}^{0}\right]_{L}=0, \\
& \sum_{\beta=1}^{2 N}\left[\bar{f}_{n} f_{m} S_{\beta}^{0}\right]_{R}\left[\bar{f}_{p} f_{q} S_{\beta}^{0}\right]_{R}=0,
\end{aligned}
$$

where $f=u$ (for up-quarks) or $f=d$ (for down-quarks). ${ }^{6}$

To derive the sum rules above, we provide details on one of the derivations.

$$
\begin{aligned}
\sum_{\beta=1}^{2 N}\left[\bar{u}_{n} u_{m} S_{\beta}^{0}\right]_{L}\left[\bar{u}_{p} u_{q} S_{\beta}^{0}\right]_{L} & =\sum_{\beta=1}^{2 N} \sum_{a=1}^{N} \sum_{b=1}^{N}\left(N_{u}^{\dagger(a)}\right)_{n m} B_{a \beta}\left(N_{u}^{\dagger(b)}\right)_{p q} B_{b \beta} \\
& =\sum_{a=1}^{N} \sum_{b=1}^{N}\left(N_{u}^{\dagger(a)}\right)_{n m}\left(N_{u}^{\dagger(b)}\right)_{p q} \sum_{\beta=1}^{2 N} B_{a \beta} B_{b \beta} \\
& =\sum_{a=1}^{N} \sum_{b=1}^{N}\left(N_{u}^{\dagger(a)}\right)_{n m}\left(N_{u}^{\dagger(b)}\right)_{p q}\left(B B^{T}\right)_{a b}=0,
\end{aligned}
$$

where the last equality is a consequence of eq. (2.30). There are numerous other cases that

\footnotetext{
${ }^{6}$ The sum rules exhibited in eqs. (3.12) and (3.13), in the special case of $n=m=p=q$, have been obtained previously in ref. [22].
} 
yields a factor $B B^{T}$ as above and thus produce a similar sum rule. For example,

$$
\begin{aligned}
& \sum_{\beta=1}^{2 N}\left[\bar{u}_{n} u_{m} S_{\beta}^{0}\right]_{L}\left[\bar{d}_{p} d_{q} S_{\beta}^{0}\right]_{R}=0, \\
& \sum_{\beta=1}^{2 N}\left[\bar{u}_{n} u_{m} S_{\beta}^{0}\right]_{R}\left[\bar{d}_{p} d_{q} S_{\beta}^{0}\right]_{L}=0 .
\end{aligned}
$$

Furthermore,

$$
\begin{aligned}
\sum_{q=1}^{3}\left[\bar{d}_{n} u_{q} S_{b}^{-}\right]_{L}\left[\bar{u}_{q} d_{m} S_{a}^{+}\right]_{R} & =\left(N_{d}^{\dagger(b)} N_{d}^{(a)}\right)_{n m} \\
\sum_{a=1}^{N} \sum_{q=1}^{3}\left[\bar{d}_{n} u_{q} S_{a}^{-}\right]_{L}\left[\bar{u}_{q} d_{m} S_{a}^{+}\right]_{R} & =\frac{1}{2} \sum_{\beta=1}^{2 N} \sum_{q=1}^{3}\left[\bar{d}_{n} d_{q} S_{\beta}^{0}\right]_{L}\left[\bar{d}_{q} d_{m} S_{\beta}^{0}\right]_{R}=\sum_{a=1}^{N}\left(N_{d}^{\dagger(a)} N_{d}^{(a)}\right)_{n m} \\
\sum_{a=1}^{N} \sum_{q=1}^{3}\left[\bar{u}_{n} d_{q} S_{a}^{+}\right]_{L}\left[\bar{d}_{q} u_{m} S_{a}^{-}\right]_{R} & =\frac{1}{2} \sum_{\beta=1}^{2 N} \sum_{q=1}^{3}\left[\bar{u}_{n} u_{q} S_{\beta}^{0}\right]_{L}\left[\bar{u}_{q} u_{m} S_{\beta}^{0}\right]_{R}=\sum_{a=1}^{N}\left(N_{u}^{\dagger(a)} N_{u}^{(a)}\right)_{n m} .
\end{aligned}
$$

Combining the parameterization of the Yukawa Lagrangian presented here with the parameterization of the scalar sector in ref. [7], we find

$$
\begin{aligned}
& \sum_{\beta=1}^{2 N}\left[\bar{f}_{n} f_{m} S_{\beta}^{0}\right]_{L}\left[Z_{\mu} S_{\beta}^{0} S_{\alpha}^{0}\right]=-i\left[\bar{f}_{n} f_{m} S_{\alpha}^{0}\right]_{L}, \\
& \sum_{\beta=1}^{2 N}\left[Z_{\mu} S_{\alpha}^{0} S_{\beta}^{0}\right]\left[\bar{f}_{n} f_{m} S_{\beta}^{0}\right]_{R}=-i\left[\bar{f}_{n} f_{m} S_{\alpha}^{0}\right]_{R} .
\end{aligned}
$$

We also observe that

$$
\begin{aligned}
& \sum_{\beta=1}^{2 N}\left[\bar{f}_{n} f_{m} S_{\beta}^{0}\right]_{L}\left[V_{\mu} V_{\nu} S_{\beta}^{0}\right]=-\left(D_{f}\right)_{n m} \\
& \sum_{\beta=1}^{2 N}\left[V_{\mu} V_{\nu} S_{\beta}^{0}\right]\left[\bar{f}_{n} f_{m} S_{\beta}^{0}\right]_{R}=-\left(D_{f}\right)_{n m}
\end{aligned}
$$

where, as before, $V_{\mu} V_{\nu}=Z_{\mu} Z_{\nu}, W_{\mu}^{+} W_{\nu}^{-}$and $f=u, d$. We find it useful to write certain sum rules that arise from the fact that the CKM matrix is unitary. For example,

$$
\begin{gathered}
\sum_{p, a}\left[\bar{u}_{n} d_{p} S_{a}^{+}\right]_{L}\left[W_{\mu}^{+} S_{\beta}^{0} S_{a}^{-}\right]\left(V^{\dagger}\right)_{p m}=\left[\bar{u}_{n} u_{m} S_{\beta}^{0}\right]_{L}, \\
-\sum_{p, a}\left(V^{\dagger}\right)_{n p}\left[\bar{u}_{p} d_{m} S_{a}^{+}\right]_{R}\left[W_{\mu}^{+} S_{\beta}^{0} S_{a}^{-}\right]=\left[\bar{d}_{n} d_{m} S_{\beta}^{0}\right]_{R},
\end{gathered}
$$




$$
\begin{aligned}
\sum_{p, a} V_{n p}\left[\bar{d}_{p} u_{m} S_{a}^{-}\right]_{R}\left[W_{\mu}^{-} S_{\beta}^{0} S_{a}^{+}\right] & =\left[\bar{u}_{n} u_{m} S_{\beta}^{0}\right]_{R}, \\
-\sum_{p, a}\left[\bar{d}_{n} u_{p} S_{a}^{-}\right]_{L}\left[W_{\mu}^{-} S_{\beta}^{0} S_{a}^{+}\right] V_{p m} & =\left[\bar{d}_{n} d_{m} S_{\beta}^{0}\right]_{L} .
\end{aligned}
$$

We have derived the sum rules above directly from the Lagrangian. One can also obtain these sum rules from unitarity bounds. Some sum rules were written for a general model in eq. (3.4) and eq. (3.7) of ref. [20]. In appendix A we show explicitly the derivation of those sum rules based on the cancellation of bad high energy behavior in $2 \rightarrow 2$ scattering processes, in the case of the most general NHDM with fermions. Note that in contrast to the results of section $\mathrm{V}$ of ref. [20], the sum rules exhibited in table 1 and in eqs. (3.20)-(3.27) have been derived under the assumption of multiple quark family generations.

\section{A critical constraint from perturbativity}

The sum rules obtained in section 3 can be used to uncover intimate relations between the structure of Yukawa couplings and the scalar/gauge couplings. As an illustration, we start by observing that eq. (2.32) can be rewritten as,

$$
\delta_{\beta \gamma}+i A_{\beta \gamma}=\sum_{a=1}^{N} B_{a \beta}^{*} B_{a \gamma} .
$$

Setting $\beta=\gamma=2$ and noting that the matrix $A$ is antisymmetric, we get

$$
1=\sum_{a=1}^{N}\left|B_{a 2}\right|^{2} .
$$

Thus, $\left|B_{12}\right|$ must be smaller than one. Moreover, we know from eqs. (2.35), (2.36) and (3.2) that $B_{12}=-\left[V V h_{125}\right]$, where we have assumed that the lowest lying neutral scalar coincides with the one found with $125 \mathrm{GeV}$ at LHC (recall that $a=1$ refers to the neutral would-be Goldstone boson, while $a=2$ refers to the lowest lying massive neutral mass eigenstate). Therefore, one may parameterize

$$
\left|\left[V V h_{125}\right]\right|^{2}=\left|B_{12}\right|^{2}=s_{\bar{\beta}-\bar{\alpha}}^{2},
$$

where, henceforth $s_{\theta}, c_{\theta}$, and $t_{\theta}$ represent the sine, cosine, and tangent of any angle $\theta$ that appears in the subscript. ${ }^{7}$ Although reminiscent of the notation in the real $2 \mathrm{HDM}$, the definition of $s_{\bar{\beta}-\bar{\alpha}}^{2}$ in eq. (4.3) is completely general. Since the value of [VVh $\left.h_{125}\right]$ deduced from the LHC Higgs data is very close to one, we conclude that $c_{\bar{\beta}-\bar{\alpha}}^{2}$ is close to zero.

Let us now define

$$
\vec{b}=\left[B_{22}, B_{32}, \ldots, B_{N 2}\right] .
$$

\footnotetext{
${ }^{7}$ We recall in passing that this discussion implies that the coupling $\left[V V h_{125}\right]$ measured at LHC is smaller than unity in any multi-Higgs doublet model. Had $\left[V V h_{125}\right]$ been found experimentally to be larger than one, then not only the SM but any NHDM would have been excluded.
} 
Clearly, the squared-magnitude of the this vector, $|\vec{b}|^{2}=c_{\bar{\beta}-\bar{\alpha}}^{2}$, must be very close to zero. Next, we recall from eq. (2.15) that the matrix $B$ takes the neutral scalars fields from the charged Higgs basis into their mass basis. Thus, $s_{\bar{\beta}-\bar{\alpha}}^{2} \sim 1$ means that the massive neutral scalar in the first doublet of the charged Higgs basis approximately coincides with the lightest neutral scalar mass eigenstates, which is identified with the observed Higgs boson of mass $125 \mathrm{GeV}$. This is known as the alignment limit [24-32]. It occurs naturally in the decoupling limit [24], but can also arise in a parameter regime without decoupling.

We will now show that, as a consequence of approximate alignment as suggested by the precision Higgs data, the vector $\vec{b}$ - which depends exclusively on properties of the neutral scalars - must be almost orthogonal to the vectors

$$
\vec{a}^{(n m)}=\left[\left(N_{d}^{(2)}\right)_{n m},\left(N_{d}^{(3)}\right)_{n m}, \ldots,\left(N_{d}^{(N)}\right)_{n m}\right],
$$

for any choice of $m$ and $n$ (explicit reference to $n$ and $m$ will henceforth be suppressed). Indeed, in light of the Cauchy-Schwarz inequality,

$$
|\vec{a} \cdot \vec{b}|^{2} \leq|\vec{a}|^{2}|\vec{b}|^{2}=|\vec{a}|^{2} c_{\bar{\beta}-\bar{\alpha}}^{2}
$$

is suppressed by $c_{\bar{\beta}-\bar{\alpha}}^{2}$. Eq. (2.53) shows that entries in the $\sqrt{2} N_{f}^{(a)} / v$ matrix are physical [up to an overall rephasing as shown in eq. (2.52)], for they appear in the Yukawa Lagrangian expressed in terms of the scalars fields in their mass basis. Moreover, for the theory to remain perturbative, such couplings cannot exceed some reference value, which we take to be $4 \pi$. As a result

$$
|\vec{a}|^{2} \leq \sum_{a \geq 2}\left|\left(N_{d}^{(a)}\right)_{n m}\right|^{2} \leq 8 \pi^{2} v^{2}(N-1),
$$

and

$$
|\vec{a} \cdot \vec{b}|^{2} \leq 8 \pi^{2} v^{2}(N-1) c_{\bar{\beta}-\bar{\alpha}}^{2} .
$$

This shows that the alignment limit, which is initially defined based on the observed $V V h_{125}$ coupling, has a dramatic impact on the Higgs-fermion Yukawa couplings. This is one of our major results. It can be written in a more interesting fashion by setting $\beta=2$ in eq. (3.9),

$$
\left[\bar{d}_{n} d_{m} S_{2}^{0}\right]_{R}=\left(N_{d}^{1}\right)_{n m} B_{12}+\sum_{a \geq 2}\left(N_{d}^{(a)}\right)_{n m} B_{a 2} .
$$

In light of eq. (2.54), it follows that,

$$
\left[\bar{d}_{n} d_{m} h_{125}\right]_{R}-m_{n}^{d} \delta_{n m} B_{12}=\vec{a} \cdot \vec{b}
$$

is also bounded by eq. (4.8). Likewise, eq. (3.8) yields

$$
\left[\bar{d}_{n} d_{m} h_{125}\right]_{L}-m_{n}^{d} \delta_{n m} B_{12}^{*}=\left(\vec{a}^{T} \cdot \vec{b}\right)^{*},
$$

where $\vec{a}^{T(n m)}=\vec{a}^{(m n)}$ [cf. eq. (4.5)]. Similar equations hold for the up type fermions. We conclude that the couplings of the observed $125 \mathrm{GeV}$ scalar to quark pairs are approximately diagonal in the alignment limit with values that approximate the corresponding coupling of 
the SM Higgs boson. Moreover, the magnitude of the off-diagonal couplings of the $125 \mathrm{GeV}$ scalar are bounded according to eq. (4.8). Of course, this behavior is expected since the tree-level properties of $H^{0} \equiv \sqrt{2} \operatorname{Re} \Phi_{1}^{C}-v$ are precisely those of the SM Higgs boson. In the alignment limit, $H^{0}$ is an approximate mass eigenstate that is identified as the observed $125 \mathrm{GeV}$ scalar.

It is instructive to apply eq. (4.10) in the case of the so-called complex two Higgs doublet model (C2HDM) (see, e.g., refs. [33-39]). A recent analysis was performed in [40], introducing the public C2HDM_HDECAY code for the HDECAY program [41], as well as all the corresponding Feynman rules. ${ }^{8}$ Using eq. (B.12) of ref. [7], we find

$$
\left[\bar{d}_{n} d_{m} h_{125}\right]_{R}+m_{n}^{d} \delta_{n m} s_{\beta-\alpha} c_{2}=\left(N_{d}^{(2)}\right)_{n m}\left(-c_{\beta-\alpha} c_{2}+i s_{2}\right) .
$$

In the C2HDM there are three mixing angles $\left(\alpha_{1}, \alpha_{2}\right.$, and $\left.\alpha_{3}\right) ; c_{2} \equiv \cos \alpha_{2}$ and similarly for others; while we define $\alpha_{1}=\alpha+\pi / 2$, in order to make contact between $\alpha_{1}$ as employed in the $\mathrm{C} 2 \mathrm{HDM}$ and the angle $\alpha$ used in its real 2HDM limit. In the notation used here, the $h_{125} V V$ coupling is given by $c_{2} \cos \left(\alpha_{1}-\beta\right)=-c_{2} s_{\beta-\alpha}$, which corresponds to $s_{\bar{\beta}-\bar{\alpha}}$ used in eq. (4.3). For the $h_{125} V V$ coupling to be close to unity, $s_{2}$ must be close to zero (i.e., a small CP-violating angle), and $c_{\beta-\alpha}$ must also be close to zero, making both terms on the right-hand-side of eq. (4.12) close to zero. Consequently, the real part of $\left[\bar{d}_{n} d_{m} h_{125}\right]_{R}$ must lie close to its SM value and its imaginary part must be close to zero. ${ }^{9}$ Eq. (4.12) for the C2HDM, and more generally eq. (4.10) in the case of the NHDM can also be used to generalize the results presented recently in ref. [42].

\section{Conclusions}

Although the Standard Model employs a Higgs sector consisting of a hypercharge-one, doublet of scalar fields, the generational structure of the fermionic sector invites us to consider the possibility that the Higgs sector of the Standard Model is also non-minimal, consisting of $N$ Higgs doublets. Without prior knowledge of $N$, it is useful to analyze the NHDM in the case of general $N$. In a previous paper [7], we examined the physical parameterization, sum rules and unitarity bounds of the bosonic sector of the NHDM. We were able to provide an elegant formulation of the NHDM by exploiting the charged Higgs basis, where the neutral scalar field vacuum expectation value resides entirely in one of the $N$ scalar doublet fields, and each of the remaining $N-1$ scalar doublet fields contains a physical (mass-eigenstate) charged Higgs field. In this formulation, many of the purely bosonic couplings of the model can be expressed entirely in terms of an $N \times 2 N$ matrix $B$.

This paper extends the results of ref. [7] to include the most general Higgs-fermion Yukawa couplings. We have shown that in addition to $B$, one must introduce a pair of $N-1$ complex $3 \times 3$ matrices (one for up-type and one for down-type), along with the diagonal up and down-type quark mass matrices in order to fully parameterize the Higgsquark Yukawa interactions. Using these parameters, we have derived a set of sum rules

\footnotetext{
${ }^{8}$ See the webpage at http://porthos.tecnico.ulisboa.pt/arXiv/C2HDM/.

${ }^{9}$ Despite the bounds on $s_{2}$ and contrary to popular belief, one can still have dominant CP-violating couplings to some fermions, even when the bounds from electric dipole moments are taken into account [38-40].
} 
that involve the Higgs-fermion interactions. Some of these sum rules exclusively involve the Yukawa couplings, whereas others involve products of Yukawa couplings and gauge/Higgs couplings. Several of these sum rules have not appeared previously in the literature.

In the charged Higgs basis, the tree-level couplings of the neutral CP-even component of the scalar doublet [denoted by $H^{0}$ in eq. (2.14)] that contains the entire neutral scalar field vacuum expectation value correspond precisely to those of the SM Higgs boson. In general, $H^{0}$ is not a mass-eigenstate due to the mixing of this field with the other neutral scalar fields of the NHDM. However, if $H^{0}$ is an approximate mass eigenstate, then the Higgs sector is said to exhibit approximate alignment, since the corresponding mass eigenstate is approximately aligned in field space with the neutral Higgs vacuum expectation value. The alignment limit can be conveniently defined by exploiting the sum rule satisfied by the $V V$ couplings to the neutral scalars (where $V V=W^{+} W^{-}$or $Z Z$ ). We are then able to show the corresponding impact of the alignment limit on the Higgs-fermion couplings.

Of course, the sum rules governing the Higgs-fermion couplings of the NHDM, while constraining the model in interesting ways, do not address the phenomenological challenge presented by the near absence of flavor-changing neutral currents in the experimental data. Without further model constraints, either via fine-tuning of couplings or by the imposition of additional symmetries, the generic NHDM will exhibit significant tree-level flavor changing neutral currents mediated by neutral Higgs exchange, in conflict with experimental observations. Addressing this challenge will be the subject of a future publication.

\section{Acknowledgments}

The work of M.P.B, J.C.R. and J.P.S. is supported in part by the Portuguese Fundação para a Ciência e Tecnologia (FCT) under contracts CERN/FIS-NUC/0010/2015, PTDC/FISPAR/29436/2017 and UID/FIS/00777/2013. H.E.H. is supported in part by the U.S. Department of Energy grant number DE-SC0010107, and in part by the grant H2020MSCA-RISE-2014 No. 645722 (NonMinimalHiggs).

\section{A Generalized sum rules}

\section{A.1 Notation and conventions}

In order to obtain the sum rules of section III of ref. [20], in particular their equations (3.3), (3.4) and (3.7), it is convenient to adopt their conventions for the Feynman rules,

$$
\begin{aligned}
V_{a}^{\alpha} V_{b}^{\beta} V_{c}^{\gamma} & : i g_{a b c}\left[\left(p_{a}-p_{b}\right)^{\gamma}+\left(p_{b}-p_{c}\right)^{\alpha}+\left(p_{c}-p_{a}\right)^{\beta}\right] \equiv i g_{a b c} \Gamma^{\alpha \beta \gamma}\left(p_{a}, p_{b}, p_{c}\right) \\
V_{a}^{\alpha} V_{c}^{\beta} \phi_{i} & : i g_{a b i} g^{\alpha \beta} \\
V_{a}^{\alpha} \phi_{i} \phi_{j} & : i g_{a i j}\left(p_{i}-p_{j}\right)^{\alpha} \\
V_{a}^{\alpha} \bar{f}_{m} f_{n} & : i \gamma^{\alpha}\left(g_{a m n}^{L} P_{L}+g_{a m n}^{R} P_{R}\right) \\
\phi_{i} \bar{f}_{m} f_{n} & : i\left(g_{i m n}^{L} P_{L}+g_{i m n}^{R} P_{R}\right)
\end{aligned}
$$



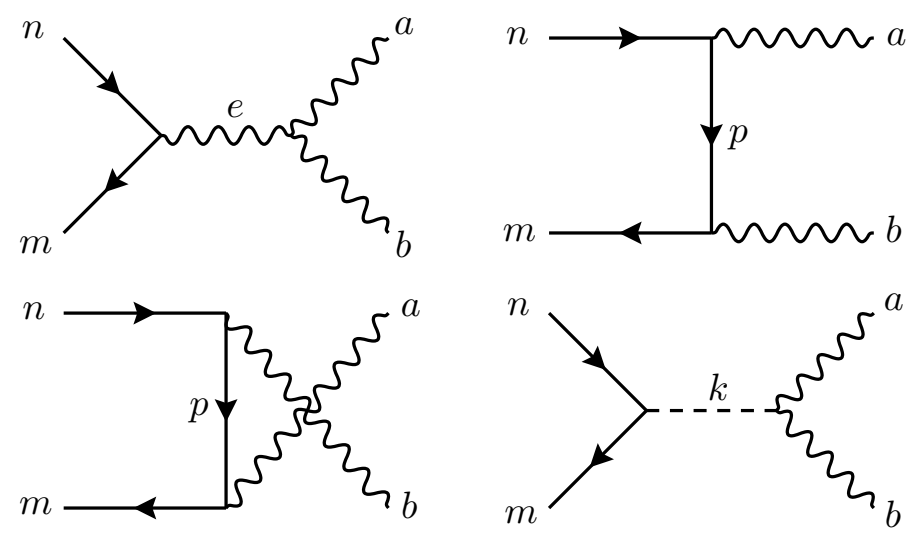

Figure 1. Diagrams for the scattering $f_{n}+\bar{f}_{m} \rightarrow V_{a} V_{b}$.

with all momenta incoming. Here $f, V$, and $\phi$ stand for fermions, gauge bosons, and scalars, respectively, and $P_{R, L} \equiv \frac{1}{2}\left(1 \pm \gamma_{5}\right)$ are the usual chiral projection operators. We will use lowercase $m_{n}$ for the mass of the fermion $f_{n}$, and uppercase $M_{a}$ for the mass of the gauge boson $V_{a}$.

\section{A.2 FFV $V$ sum rules}

\section{A.2.1 The amplitudes}

The diagrams contributing to the scattering $f_{n}\left(p_{1}\right)+\bar{f}_{m}\left(p_{2}\right) \rightarrow V_{a}\left(p_{3}\right)+V_{b}\left(p_{4}\right)$ are exhibited in figure 1. In an obvious notation we will name the amplitudes according to Mandelstam variables channel $(s, t$ or $u)$ and by the particle being exchanged. We then obtain,

$$
\begin{aligned}
\mathcal{M}_{s}^{A}=(- & i)\left(i g_{a b e}\right) \Gamma_{\alpha \beta \nu}\left(-p_{4},-p_{3}, p_{1}+p_{2}\right) i \bar{f}_{m}\left(p_{2}\right) \gamma_{\mu}\left(g_{e m n}^{L} P_{L}+g_{e m n}^{R} P_{R}\right) f_{n}\left(p_{1}\right) \\
& \times(-i) \frac{\left[g^{\mu \nu}-\left(p_{1}+p_{2}\right)^{\mu}\left(p_{1}+p_{2}\right)^{\nu} / M_{e}^{2}\right]}{s-M_{e}^{2}} \epsilon^{\alpha}\left(p_{3}\right) \epsilon^{\beta}\left(p_{4}\right), \\
\mathcal{M}_{t}^{f}=(- & i)(i)^{3} \bar{f}_{m}\left(p_{2}\right) \gamma_{\beta}\left(g_{b m p}^{L} P_{L}+g_{b m p}^{R} P_{R}\right)\left(\not p_{1}-\not p_{3}+m_{p}\right) \gamma_{\alpha}\left(g_{a p n}^{L} P_{L}+g_{a p n}^{R} P_{R}\right) f_{n}\left(p_{1}\right) \\
& \quad \times \frac{1}{t-m_{p}^{2}} \epsilon^{\alpha}\left(p_{3}\right) \epsilon^{\beta}\left(p_{4}\right), \\
\mathcal{M}_{u}^{f}=(-i)(i)^{3} \bar{f}_{m}\left(p_{2}\right) \gamma_{\alpha}\left(g_{a m p}^{L} P_{L}+g_{a m p}^{R} P_{R}\right)\left(\not p_{1}-\not p_{3}+m_{p}\right) \gamma_{\beta}\left(g_{b p n}^{L} P_{L}+g_{b p n}^{R} P_{R}\right) f_{n}\left(p_{1}\right) & \\
& \quad \times \frac{1}{u-m_{p}^{2}} \epsilon^{\alpha}\left(p_{3}\right) \epsilon^{\beta}\left(p_{4}\right), \\
\mathcal{M}_{s}^{\phi}= & (-i)(i)^{3} g_{a b k} \bar{f}_{m}\left(p_{2}\right)\left(g_{k m n}^{L} P_{L}+g_{k m n}^{R} P_{R}\right) f_{n}\left(p_{1}\right) \frac{g_{\alpha \beta}}{s-m_{k}^{2}} \epsilon^{\alpha}\left(p_{3}\right) \epsilon^{\beta}\left(p_{4}\right) .
\end{aligned}
$$

\section{A.2.2 The high energy limit}

When the gauge bosons are longitudinally polarized the diagrams of figure 1 grow with energy for large center of mass energy $\sqrt{s}$. The worst behavior comes from the first three diagrams that grow like $E^{2}$ while the fourth diagram grows like $E m_{f}$. To see this one has 
to use the expression for the polarization vector for the longitudinal case, which is given by

$$
\epsilon_{L}=(\gamma \beta, \gamma \vec{\beta} / \beta) \simeq \frac{p^{\mu}}{M}+\mathcal{O}\left(\frac{1}{\gamma^{2}} \frac{E}{M}\right) .
$$

To determine the coefficients of the high energy behavior [see eq. (A.20) below] we cannot use the approximate expression in the right-hand side of eq. (A.7) for all the diagrams because we would then lose contributions that modify the $E m_{f}$ terms. Hence, we should employ consistently the definitions of the left-hand side and expand the result in powers of $s, t$ or $u$. As an example, for the gauge boson $V_{a}$, we have

$$
\epsilon_{a}^{L}=\left(\gamma_{a} \beta_{a}, \gamma_{a} \vec{\beta}_{a} / \beta_{a}\right), \beta_{a}=\frac{\sqrt{E_{a}^{2}-M_{a}^{2}}}{E_{a}}, \gamma_{a}=\frac{1}{\sqrt{1-\beta_{a}^{2}}}, E_{a}=\frac{s+M_{a}^{2}-M_{b}^{2}}{2 \sqrt{s}},
$$

and similarly for the other particles. Next we use the kinematics for the process,

$$
f\left(p_{1}\right)+\bar{f}\left(p_{2}\right) \rightarrow V_{a}\left(p_{3}\right)+V_{b}\left(p_{4}\right),
$$

to write

$$
\begin{array}{ll}
p_{1}=\left(E_{n}, 0,0, \beta_{n} E_{n}\right), & p_{2}=\left(E_{m}, 0,0,-\beta_{m} E_{m}\right), \\
p_{3}=\left(E_{a}, \beta_{a} E_{a} \sin \theta, 0, \beta_{a} E_{a} \cos \theta\right), & p_{4}=\left(E_{b},-\beta_{b} E_{b} \sin \theta, 0,-\beta_{b} E_{b} \cos \theta\right), \\
\epsilon_{a}^{L}=\left(\gamma_{a} \beta_{a}, \gamma_{a} \sin \theta, 0, \gamma_{a} \cos \theta\right), & \epsilon_{b}^{L}=\left(\gamma_{b} \beta_{b},-\gamma_{b} \sin \theta, 0,-\gamma_{b} \cos \theta\right) .
\end{array}
$$

We then use these expressions to evaluate all the amplitudes. In the end we substitute $\cos \theta$ in terms of the Mandelstam variable $t$, through the relation,

$$
\cos \theta=\frac{t-m_{n}^{2}-M_{a}^{2}+2 E_{n} E_{a}}{2 E_{n} E_{a} \beta_{n} \beta_{a}} .
$$

At this point all the amplitudes are expressed in terms of the Mandelstam variables and the masses. As the Mandelstam variables are not independent, we can still use the relation,

$$
s+t+u=m_{n}^{2}+m_{m}^{2}+M_{a}^{2}+M_{b}^{2},
$$

to express the result in terms of just two independent variables. Next we want to isolate the terms that grow with $E^{2}$ and $E m_{f}$. To achieve this we introduce the scaling

$$
s \rightarrow s / x, \quad t \rightarrow t / x, \quad u \rightarrow u / x,
$$

and make an expansion for small $x$. This would be enough for the amplitudes without fermions, but here we have the additional complication of having strings like

$$
\bar{f}\left(p_{2}\right) \cdots f\left(p_{1}\right) .
$$

Since we want to isolate the coefficients of these structures, and as the spinors grow like $E^{1 / 2}$, we also employ the scaling

$$
\bar{f}\left(p_{2}\right) \cdots f\left(p_{1}\right) \rightarrow \frac{1}{\sqrt{x}} \bar{f}\left(p_{2}\right) \cdots f\left(p_{1}\right) .
$$


There is one final complication. Since we wish to have independent spinor structures, we shall use the Dirac equation between spinors. But then we have a problem for structures like

$$
\bar{f}\left(p_{2}\right) \cdots \gamma_{\alpha} \cdots f\left(p_{1}\right) \epsilon^{\alpha}
$$

We have verified that for this case it is enough to use the first term on the right-hand side of eq. (A.7). That is, we will make the replacement

$$
\gamma^{\alpha} \epsilon_{\alpha}(p) \rightarrow \frac{1}{\sqrt{x}} \gamma^{\alpha} \frac{p_{\alpha}}{M}
$$

The terms that grow as $E^{2}$ are the coefficients of $x^{-1}$ and the terms that grow as $E m_{f}$ are the coefficients of $x^{-1 / 2}$. Therefore we can write for each amplitude

$$
\begin{aligned}
M_{i}= & \bar{f}\left(p_{2}\right) \not p_{3} P_{L} f\left(p_{1}\right) A_{i}^{L} x^{-1}+\bar{f}\left(p_{2}\right) \not p_{3} P_{R} f\left(p_{1}\right) A_{i}^{R} x^{-1} \\
& +\bar{f}\left(p_{2}\right) P_{L} f\left(p_{1}\right) B_{i}^{L} x^{-1 / 2}+\bar{f}\left(p_{2}\right) P_{R} f\left(p_{1}\right) B_{i}^{R} x^{-1 / 2}+\text { constant },
\end{aligned}
$$

where we have assumed energy-momentum conservation. Eq. (A.20) has been obtained by using FeynCalc and Mathematica for the Lorentz and Dirac algebra and series expansion, respectively.

The $\boldsymbol{E}^{\mathbf{2}}$ terms. The first three diagrams in figure 1 yield terms that grow like $E^{2}$. To simplify the expressions, we redefine the coefficients

$$
\hat{A}_{i}=A_{i} M_{a} M_{b}
$$

The corresponding $\hat{A}_{i}$ coefficients are given in table 2 . Since the sum of these coefficients has to vanish, we end up with two sum rules,

$$
\begin{aligned}
& \sum_{p}\left[g_{a p n}^{L} g_{b m p}^{L}-g_{a m p}^{L} g_{b p n}^{L}\right]=\sum_{e} g_{a b e} g_{e m n}^{L}, \\
& \sum_{p}\left[g_{a p n}^{R} g_{b m p}^{R}-g_{a m p}^{R} g_{b p n}^{R}\right]=\sum_{e} g_{a b e} g_{e m n}^{R} .
\end{aligned}
$$

These relations are the sum rules in eq. (3.3) of ref [20]. The cancellation of the terms which grow as $E^{2}$ is guaranteed by the gauge group structure of the fermion representations, as shown by Llewellyn Smith [43] (see also [44, 45]). So, these sum rules must hold in any spontaneously broken gauge theory.

The $\boldsymbol{E}$ terms. Having shown that a spontaneously broken gauge theory assures that the worst high energy behavior cancels, we move to the terms that grow as a single power of $E$. Here the gauge invariance of the theory is not sufficient to guarantee cancellation of the bad high energy behavior, and we obtain constraints on the gauge boson couplings to scalars.

For convenience we again define,

$$
\hat{B}_{i} \equiv M_{a} M_{b} B_{i} .
$$




\begin{tabular}{|c|c|c|}
\hline Diagram & $\hat{A}_{i}^{L}$ & $\hat{A}_{i}^{R}$ \\
\hline $\mathcal{M}_{s}^{A}$ & $g_{\text {abe }} g_{\text {emn }}^{L}$ & $g_{\text {abe }} g_{\text {emn }}^{R}$ \\
\hline $\mathcal{M}_{t}^{f}$ & $-g_{\text {apn }}^{L} g_{b m p}^{L}$ & $-g_{a p n}^{R} g_{b m p}^{R}$ \\
\hline $\mathcal{M}_{u}^{f}$ & $g_{a m p}^{L} g_{b p n}^{L}$ & $g_{a m p}^{R} g_{b p n}^{R}$ \\
\hline $\mathcal{M}_{s}^{\phi}$ & 0 & 0 \\
\hline
\end{tabular}

Table 2. Coefficients $\hat{A}_{i}^{L}$ and $\hat{A}_{i}^{R}$.

The results are summarized in table 3. To obtain the sum rule in eq. (3.4) of ref. [20], the sum of the coefficients $\hat{B}_{i}^{L}$ and $\hat{B}_{i}^{R}$ has to vanish separately. The follow sum rule is then obtained,

$$
\begin{aligned}
\sum_{p}\left[m_{p}\right. & \left.\left(g_{b m p}^{R} g_{a p n}^{L}+g_{a m p}^{R} g_{b p n}^{L}\right)-m_{m} g_{a p n}^{L} g_{b m p}^{L}-m_{n} g_{a m p}^{R} g_{b p n}^{R}\right] \\
& +\sum_{e}^{\prime}\left[g_{a b e}\left[\frac{M_{a}^{2}-M_{b}^{2}-M_{e}^{2}}{2 M_{e}^{2}}\right]\left(m_{n} g_{e m n}^{R}-m_{m} g_{e m n}^{L}\right)\right]=\frac{1}{2} \sum_{k} g_{a b k} g_{k m n}^{L}
\end{aligned}
$$

where $\sum_{e}{ }^{\prime}$ means that the sum only runs over massive gauge bosons. Another sum rule can be obtained from eq. (A.25) with the substitution $L \leftrightarrow R$. Eq. (A.25) is similar, but not equal, to eq. (3.4) of ref. [20]. But we can bring eq. (A.25) to the form of the sum rule of ref. [20] using eq. (A.22) to write,

$$
\begin{aligned}
& -\sum_{p} m_{m} g_{a p n}^{L} g_{b m p}^{L}=-\sum_{p} m_{m} g_{a m p}^{L} g_{b p n}^{L}-\sum_{e} g_{a b e} m_{m} g_{e m n}^{L}, \\
& -\sum_{p} m_{n} g_{a m p}^{R} g_{b p n}^{R}=-\sum_{p} m_{n} g_{a p n}^{R} g_{b m p}^{R}+\sum_{e} g_{a b e} m_{n} g_{e m n}^{R} .
\end{aligned}
$$

Now we add the last two equations to obtain

$$
\begin{aligned}
\sum_{p}\left[-m_{m} g_{a p n}^{L} g_{b m p}^{L}-m_{n} g_{a m p}^{R} g_{b p n}^{R}\right]= & \sum_{p}\left[-m_{m} g_{a m p}^{L} g_{b p n}^{L}-m_{n} g_{a p n}^{R} g_{b m p}^{R}\right] \\
& +\sum_{e}{ }^{\prime} g_{a b e}\left(m_{n} g_{\text {emn }}^{R}-m_{m} g_{\text {emn }}^{L}\right) .
\end{aligned}
$$

Finally, we substitute eq. (A.27) into eq. (A.25), to obtain

$$
\begin{aligned}
\sum_{p}\left[m_{p}\right. & \left.\left(g_{b m p}^{R} g_{a p n}^{L}+g_{a m p}^{R} g_{b p n}^{L}\right)-m_{m} g_{a m p}^{L} g_{b p n}^{L}-m_{n} g_{a p n}^{R} g_{b m p}^{R}\right] \\
& +\sum_{e}^{\prime}\left[g_{a b e}\left[\frac{M_{a}^{2}-M_{b}^{2}+M_{e}^{2}}{2 M_{e}^{2}}\right]\left(m_{n} g_{e m n}^{R}-m_{m} g_{e m n}^{L}\right)\right]=\frac{1}{2} \sum_{k} g_{a b k} g_{k m n}^{L},
\end{aligned}
$$

which is precisely the sum rule of eq. (3.4) of ref. [20]. We also obtain a similar rule by substituting $L \leftrightarrow R$. The other sum rules in eqs. (3.5) and (3.6) of ref. [20] can be derived from the above in the same way. 

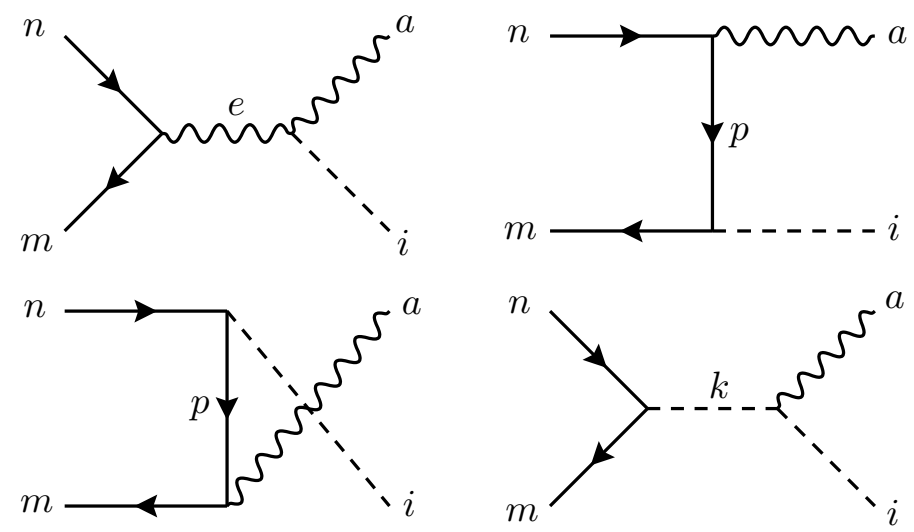

Figure 2. Diagrams for the scattering $f_{n}+\bar{f}_{m} \rightarrow V_{a} \phi_{i}$.

\begin{tabular}{|c|c|c|}
\hline Diagram & $\hat{B}_{i}^{L}$ & $\hat{B}_{i}^{R}$ \\
\hline$\frac{\mathcal{M}_{s}^{A}}{g_{a b e}\left[\frac{\left(M_{a}^{2}-M_{b}^{2}-M_{e}^{2}\right)}{2 M_{e}^{2}}\right]}$ & $g_{e m n}^{R} m_{n}-g_{\text {emn }}^{L} m_{m}$ & $g_{e m n}^{L} m_{n}-g_{e m n}^{R} m_{m}$ \\
\hline $\mathcal{M}_{t}^{f}$ & $g_{a p n}^{L} g_{b m p}^{R} m_{p}-g_{a p n}^{L} g_{b m p}^{L} m_{m}$ & $g_{a p n}^{R} g_{b m p}^{L} m_{p}-g_{a p n}^{R} g_{b m p}^{R} m_{m}$ \\
\hline $\mathcal{M}_{u}^{f}$ & $g_{b p n}^{L} g_{a m p}^{R} m_{p}-g_{a m p}^{R} g_{b p n}^{R} m_{n}$ & $g_{b p n}^{R} g_{a m p}^{L} m_{p}-g_{a m p}^{L} g_{b p n}^{L} m_{n}$ \\
\hline $\mathcal{M}_{s}^{\phi}$ & $-\frac{1}{2} g_{a b k} g_{k m n}^{L}$ & $-\frac{1}{2} g_{a b k} g_{k m n}^{R}$ \\
\hline
\end{tabular}

Table 3. Coefficients $\hat{B}_{i}$.

\section{A.3 FF $\boldsymbol{F} \phi$ sum rules}

\section{A.3.1 The amplitudes}

The diagrams contributing to the scattering $f_{n}\left(p_{1}\right)+\bar{f}_{m}\left(p_{2}\right) \rightarrow V_{a}\left(p_{3}\right)+\phi_{i}\left(p_{4}\right)$ are given in figure 2. The corresponding amplitudes are given by,

$\mathcal{M}_{s}^{A}=(-i)^{2}(i)^{2}\left(g_{\text {aie }}\right) g_{\mu \alpha} \bar{f}_{m}\left(p_{2}\right) \gamma_{\nu}\left(g_{e m n}^{L} P_{L}+g_{e m n}^{R} P_{R}\right) f_{n}\left(p_{1}\right) \frac{\left[g^{\mu \nu}-\left(p_{1}+p_{2}\right)^{\mu}\left(p_{1}+p_{2}\right)^{\nu} / M_{e}^{2}\right]}{s-M_{e}^{2}} \epsilon^{\alpha}\left(p_{3}\right)$,

$\mathcal{M}_{t}^{f}=(-i)(i)^{3} \bar{f}_{m}\left(p_{2}\right)\left(g_{i m p}^{L} P_{L}+g_{i m p}^{R} P_{R}\right)\left(\not p_{1}-\not p_{3}+m_{p}\right) \gamma_{\alpha}\left(g_{a p n}^{L} P_{L}+g_{a p n}^{R} P_{R}\right) f_{n}\left(p_{1}\right) \frac{1}{t-m_{p}^{2}} \epsilon^{\alpha}\left(p_{3}\right)$,

$\mathcal{M}_{u}^{f}=(-i)(i)^{3} \bar{f}_{m}\left(p_{2}\right) \gamma_{\alpha}\left(g_{a m p}^{L} P_{L}+g_{a m p}^{R} P_{R}\right)\left(\not p_{1}-\not p_{3}+m_{p}\right)\left(g_{i p n}^{L} P_{L}+g_{i p n}^{R} P_{R}\right) f_{n}\left(p_{1}\right) \frac{1}{u-m_{p}^{2}} \epsilon^{\alpha}\left(p_{3}\right)$,

$\mathcal{M}_{s}^{\phi}=(-i)(i)^{3} g_{a i k}\left(-p_{4}-p_{1}-p_{2}\right)_{\alpha} \bar{f}_{m}\left(p_{2}\right)\left(g_{k m n}^{L} P_{L}+g_{k m n}^{R} P_{R}\right) f_{n}\left(p_{1}\right) \frac{1}{s-m_{k}^{2}} \epsilon^{\alpha}\left(p_{3}\right)$.

\section{A.3.2 The high energy limit}

In this case there are no divergent $E^{2}$ terms. The coefficients of $\hat{B}_{i}^{L}$ and $\hat{B}_{i}^{R}$ are summarized in table 4. Again we employed a definition similar to eq. (A.24),

$$
\hat{B}_{i}^{L, R} \equiv M_{a} B_{i}^{L, R} .
$$




\begin{tabular}{|c|c|c|}
\hline Diagram & $\hat{B}_{i}^{L}$ & $\hat{B}_{i}^{R}$ \\
\hline $\mathcal{M}_{s}^{A}$ & $-\frac{1}{2 M_{e}^{2}} g_{a e i}\left(m_{n} g_{e m n}^{R}-m_{m} g_{e m n}^{L}\right)$ & $-\frac{1}{2 M_{e}^{2}} g_{a e i}\left(m_{n} g_{e m n}^{L}-m_{m} g_{e m n}^{R}\right)$ \\
\hline $\mathcal{M}_{t}^{f}$ & $g_{a p n}^{L} g_{i m p}^{L}$ & $g_{a p n}^{R} g_{i m p}^{R}$ \\
\hline $\mathcal{M}_{u}^{f}$ & $-g_{i p n}^{L} g_{a m p}^{R}$ & $-g_{i p n}^{R} g_{a m p}^{L}$ \\
\hline $\mathcal{M}_{s}^{\phi}$ & $g_{a i k} g_{k m n}^{L}$ & $g_{a i k} g_{k m n}^{R}$ \\
\hline
\end{tabular}

Table 4. Coefficients $\hat{B}_{i}^{L, R}$.

Since the sum of the coefficients has to vanish, we obtain the sum rule,

$$
\sum_{e}^{\prime} \frac{1}{2 M_{e}^{2}} g_{a e i}\left(m_{n} g_{e m n}^{R}-m_{m} g_{e m n}^{L}\right)-\sum_{k} g_{a i k} g_{k m n}^{L}=\sum_{p}\left(g_{a p n}^{L} g_{i m p}^{L}-g_{a m p}^{R} g_{i p n}^{L}\right)
$$

in agreement with eq. (3.7) of ref. [20]. We also obtain a similar sum rule with the interchange $L \leftrightarrow R$.

Open Access. This article is distributed under the terms of the Creative Commons Attribution License (CC-BY 4.0), which permits any use, distribution and reproduction in any medium, provided the original author(s) and source are credited.

\section{References}

[1] ATLAS collaboration, Observation of a new particle in the search for the Standard Model Higgs boson with the ATLAS detector at the LHC, Phys. Lett. B 716 (2012) 1 [arXiv:1207.7214] [INSPIRE].

[2] CMS collaboration, Observation of a new boson at a mass of $125 \mathrm{GeV}$ with the CMS experiment at the LHC, Phys. Lett. B 716 (2012) 30 [arXiv:1207.7235] [INSPIRE].

[3] ATLAS and CMS collaborations, Measurements of the Higgs boson production and decay rates and constraints on its couplings from a combined ATLAS and CMS analysis of the LHC pp collision data at $\sqrt{s}=7$ and $8 \mathrm{TeV}$, JHEP 08 (2016) 045 [arXiv:1606.02266] [INSPIRE].

[4] T.D. Lee, A Theory of Spontaneous T Violation, Phys. Rev. D 8 (1973) 1226 [inSPIRE].

[5] S.L. Glashow and S. Weinberg, Natural Conservation Laws for Neutral Currents, Phys. Rev. D 15 (1977) 1958 [INSPIRE].

[6] E.A. Paschos, Diagonal Neutral Currents, Phys. Rev. D 15 (1977) 1966 [inSPIRE].

[7] M.P. Bento, H.E. Haber, J.C. Romão and J.P. Silva, Multi-Higgs doublet models: physical parametrization, sum rules and unitarity bounds, JHEP 11 (2017) 095 [arXiv:1708.09408] [INSPIRE].

[8] J.C. Romao and J.P. Silva, A resource for signs and Feynman diagrams of the Standard Model, Int. J. Mod. Phys. A 27 (2012) 1230025 [arXiv:1209.6213] [INSPIRE].

[9] F.J. Botella and J.P. Silva, Jarlskog-like invariants for theories with scalars and fermions, Phys. Rev. D 51 (1995) 3870 [hep-ph/9411288] [INSPIRE]. 
[10] G.C. Branco, L. Lavoura and J.P. Silva, CP Violation, Oxford University Press, Oxford U.K. (1999) [Int. Ser. Monogr. Phys. 103 (1999) 1] [INSPIRE].

[11] C.C. Nishi, The Structure of potentials with N Higgs doublets, Phys. Rev. D 76 (2007) 055013 [arXiv:0706.2685] [INSPIRE].

[12] H.E. Haber, O.M. Ogreid, P. Osland and M.N. Rebelo, Symmetries and Mass Degeneracies in the Scalar Sector, arXiv: 1808.08629 [INSPIRE].

[13] F.D. Murnaghan, The Unitary and Rotation Groups, Spartan Books, Washington, D.C. U.S.A. (1962).

[14] H.E. Haber and D. O'Neil, Basis-independent methods for the two-Higgs-doublet model. II. The Significance of tan $\beta$, Phys. Rev. D 74 (2006) 015018 [Erratum ibid. D 74 (2006) 059905] [hep-ph/0602242] [INSPIRE].

[15] W. Grimus and L. Lavoura, Soft lepton flavor violation in a multi Higgs doublet seesaw model, Phys. Rev. D 66 (2002) 014016 [hep-ph/0204070] [INSPIRE].

[16] W. Grimus, L. Lavoura, O.M. Ogreid and P. Osland, A Precision constraint on multi-Higgs-doublet models, J. Phys. G 35 (2008) 075001 [arXiv:0711.4022] [INSPIRE].

[17] W. Grimus, L. Lavoura, O.M. Ogreid and P. Osland, The Oblique parameters in multi-Higgs-doublet models, Nucl. Phys. B 801 (2008) 81 [arXiv:0802.4353] [INSPIRE].

[18] W. Grimus and H. Neufeld, Radiative Neutrino Masses in an $\mathrm{SU}(2) \times \mathrm{U}(1)$ Model, Nucl. Phys. B 325 (1989) 18 [INSPIRE].

[19] H.G.J. Veltman, The Equivalence Theorem, Phys. Rev. D 41 (1990) 2294 [inSPIRE].

[20] J.F. Gunion, H.E. Haber and J. Wudka, Sum rules for Higgs bosons, Phys. Rev. D 43 (1991) 904 [INSPIRE].

[21] B. Grinstein, C.W. Murphy, D. Pirtskhalava and P. Uttayarat, Theoretical Constraints on Additional Higgs Bosons in Light of the 126 GeV Higgs, JHEP 05 (2014) 083 [arXiv: 1401.0070] [INSPIRE].

[22] I.F. Ginzburg, Multi-Higgs models. Perspectives for identification of wide set of models in future experiments at colliders in the SM-like situation, in proceedings of the 22th International Workshop on High Energy Physics and Quantum Field Theory (QFTHEP 2015), Samara, Russia, 24 June-1 July 2015, arXiv:1502.07197 [InSPIRE].

[23] J.F. Gunion, H.E. Haber, G. Kane and S. Dawson, The Higgs Hunter's Guide, Westview Press, Boulder CO U.S.A. (2000) [Front. Phys. 80 (2000) 1] [INSPIRE].

[24] J.F. Gunion and H.E. Haber, The CP conserving two Higgs doublet model: The Approach to the decoupling limit, Phys. Rev. D 67 (2003) 075019 [hep-ph/0207010] [INSPIRE].

[25] N. Craig, J. Galloway and S. Thomas, Searching for Signs of the Second Higgs Doublet, arXiv:1305.2424 [INSPIRE].

[26] H.E. Haber, The Higgs data and the Decoupling Limit, in proceedings of the 1st Toyama International Workshop on Higgs as a Probe of New Physics 2013 (HPNP2013), Toyama, Japan, 13-16 February 2013, arXiv:1401.0152 [INSPIRE].

[27] D.M. Asner et al., ILC Higgs White Paper, in proceedings of the 2013 Community Summer Study on the Future of U.S. Particle Physics: Snowmass on the Mississippi (CSS2013), Minneapolis, MN, U.S.A., 29 July-6 August 2013, arXiv:1310.0763 [INSPIRE]. 
[28] M. Carena, I. Low, N.R. Shah and C.E.M. Wagner, Impersonating the Standard Model Higgs Boson: Alignment without Decoupling, JHEP 04 (2014) 015 [arXiv:1310.2248] [INSPIRE].

[29] M. Carena, H.E. Haber, I. Low, N.R. Shah and C.E.M. Wagner, Complementarity between Nonstandard Higgs Boson Searches and Precision Higgs Boson Measurements in the MSSM, Phys. Rev. D 91 (2015) 035003 [arXiv: 1410.4969] [InSPIRE].

[30] P.S. Bhupal Dev and A. Pilaftsis, Maximally Symmetric Two Higgs Doublet Model with Natural Standard Model Alignment, JHEP 12 (2014) 024 [Erratum JHEP 11 (2015) 147] [arXiv:1408.3405] [INSPIRE].

[31] J. Bernon, J.F. Gunion, H.E. Haber, Y. Jiang and S. Kraml, Scrutinizing the alignment limit in two-Higgs-doublet models: $m_{h}=125 \mathrm{GeV}$, Phys. Rev. D 92 (2015) 075004 [arXiv: 1507.00933] [INSPIRE].

[32] J. Bernon, J.F. Gunion, H.E. Haber, Y. Jiang and S. Kraml, Scrutinizing the alignment limit in two-Higgs-doublet models. II. $m_{H}=125 \mathrm{GeV}$, Phys. Rev. D 93 (2016) 035027 [arXiv: 1511.03682] [INSPIRE].

[33] I.F. Ginzburg, M. Krawczyk and P. Osland, Two Higgs doublet models with CP-violation, in proceedings of the 10th International Conference on Supersymmetry and Unification of Fundamental Interactions (SUSY02), Seogwipo, Jeju Island, Korea, 26-30 August 2002, pp. 703-706 [hep-ph/0211371] [INSPIRE].

[34] A.W. El Kaffas, P. Osland and O.M. Ogreid, CP violation, stability and unitarity of the two Higgs doublet model, Nonlin. Phenom. Complex Syst. 10 (2007) 347 [hep-ph/0702097] [INSPIRE].

[35] A. Arhrib, E. Christova, H. Eberl and E. Ginina, CP violation in charged Higgs production and decays in the Complex Two Higgs Doublet Model, JHEP 04 (2011) 089 [arXiv: 1011.6560] [INSPIRE].

[36] A. Barroso, P.M. Ferreira, R. Santos and J.P. Silva, Probing the scalar-pseudoscalar mixing in the 125 GeV Higgs particle with current data, Phys. Rev. D 86 (2012) 015022 [arXiv: 1205.4247] [INSPIRE].

[37] D. Fontes, J.C. Romão and J.P. Silva, $h \rightarrow Z \gamma$ in the complex two Higgs doublet model, JHEP 12 (2014) 043 [arXiv: 1408.2534] [INSPIRE].

[38] D. Fontes, J.C. Romão, R. Santos and J.P. Silva, Large pseudoscalar Yukawa couplings in the complex 2HDM, JHEP 06 (2015) 060 [arXiv:1502.01720] [INSPIRE].

[39] D. Fontes, J.C. Romão, R. Santos and J.P. Silva, Undoubtable signs of CP-violation in Higgs boson decays at the LHC run 2, Phys. Rev. D 92 (2015) 055014 [arXiv:1506.06755] [INSPIRE].

[40] D. Fontes, M. Mühlleitner, J.C. Romão, R. Santos, J.P. Silva and J. Wittbrodt, The C2HDM revisited, JHEP 02 (2018) 073 [arXiv:1711.09419] [INSPIRE].

[41] A. Djouadi, J. Kalinowski and M. Spira, HDECAY: A Program for Higgs boson decays in the standard model and its supersymmetric extension, Comput. Phys. Commun. 108 (1998) 56 [hep-ph/9704448] [INSPIRE].

[42] A. Dery, C. Frugiuele and Y. Nir, Large Higgs-electron Yukawa coupling in 2HDM, JHEP 04 (2018) 044 [arXiv: 1712.04514] [INSPIRE]. 
[43] C.H. Llewellyn Smith, High-Energy Behavior and Gauge Symmetry, Phys. Lett. B 46 (1973) 233 [INSPIRE].

[44] J.M. Cornwall, D.N. Levin and G. Tiktopoulos, Derivation of Gauge Invariance from High-Energy Unitarity Bounds on the S Matrix, Phys. Rev. D 10 (1974) 1145 [Erratum ibid. D 11 (1975) 972] [INSPIRE].

[45] H.A. Weldon, The Effects of Multiple Higgs Bosons on Tree Unitarity, Phys. Rev. D 30 (1984) 1547 [INSPIRE]. 